\title{
Evaluation of methods to purify virus-like particles for metagenomic sequencing of intestinal viromes
}

\author{
Manuel Kleiner ${ }^{1,3}$, Lora V Hooper ${ }^{1,2^{*}}$ and Breck A Duerkop ${ }^{1 *}$
}

\begin{abstract}
Background: Viruses are a significant component of the intestinal microbiota in mammals. In recent years, advances in sequencing technologies and data analysis techniques have enabled detailed metagenomic studies investigating intestinal viromes (collections of bacteriophage and eukaryotic viral nucleic acids) and their potential contributions to the ecology of the microbiota. An important component of virome studies is the isolation and purification of virus-like particles (VLPS) from intestinal contents or feces. Several methods have been applied to isolate VLPS from intestinal samples, yet to our knowledge, the efficiency and reproducibility between methods have not been explored. A rigorous evaluation of methods for VLP purification is critical as many studies begin to move from descriptive analyses of virus diversity to studies striving to quantitatively compare viral abundances across many samples. Therefore, reproducible VLP purification methods which allow for high sample throughput are needed. Here we compared and evaluated four methods for VLP purification using artificial intestinal microbiota samples of known bacterial and viral composition.

Results: We compared the following four methods of VLP purification from fecal samples: (i) filtration + DNase, (ii) dithiothreitol treatment + filtration + DNase, (iii) filtration + DNase + PEG precipitation and (iv) filtration + DNase + CsCl density gradient centrifugation. Three of the four tested methods worked well for VLP purification. We observed several differences between methods related to the removal efficiency of bacterial and host DNAs and biases against specific phages. In particular the $\mathrm{CsCl}$ density gradient centrifugation method, which is frequently used for VLP purification, was most efficient in removing host derived DNA, but also showed strong discrimination against specific phages and showed a lower reproducibility of quantitative results.

Conclusions: Based on our data we recommend the use of methods (i) or (ii) for large scale studies when quantitative comparison of viral abundances across samples is required. The $\mathrm{CsCl}$ density gradient centrifugation method, while being excellently suited to achieve highly purified samples, in our opinion, should be used with caution when performing quantitative studies.
\end{abstract}

Keywords: Virus metagenomics, Viral metagenomes, Virus-like particles, Microbiome, Bacteriophage, CsCl density gradient

\section{Background}

In the last decade there has been an increasing appreciation that the intestinal microbiota of mammals has a strong influence on host metabolism, physiology and

\footnotetext{
* Correspondence: lora.hooper@utsouthwestern.edu;

breck.duerkop@utsouthwestern.edu

'Department of Immunology, University of Texas Southwestern Medical

Center, Dallas, TX 75390, USA

${ }^{2}$ The Howard Hughes Medical Institute, University of Texas Southwestern Medical Center, Dallas, TX 75390, USA

Full list of author information is available at the end of the article
}

health [1-3]. Metagenomics has enabled large scale studies of these complex microbial communities in the intestine revealing both a qualitative and quantitative picture of the phylogenetic and functional diversity of intestinal microbes [4-6]. The majority of intestinal metagenomic studies have focused on the bacterial component of the microbiota during states of health and disease. In recent years viruses, including bacteriophages (phages), from the mammalian intestine have started to receive much attention [7-9]. The contribution of phages to intestinal microbiota ecology and their potential effects on the

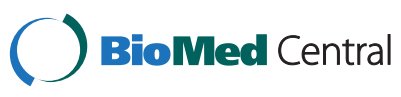

(c) 2015 Kleiner et al.; licensee Biomed Central. This is an Open Access article distributed under the terms of the Creative Commons Attribution License (http://creativecommons.org/licenses/by/4.0), which permits unrestricted use, distribution, and reproduction in any medium, provided the original work is properly credited. The Creative Commons Public Domain Dedication waiver (http://creativecommons.org/publicdomain/zero/1.0/) applies to the data made available in this article unless otherwise stated. 
mammalian host are just beginning to be elucidated [10-14].

To unravel the influence of viruses, and particularly bacteriophages, on microbiota ecology and animal host physiology and health, methods allowing quantitative comparison of virus diversity, abundance and function across samples are needed. Sequencing of viromes (metagenomes of virus-like particles, VLPs) is one method that enables such quantitative comparisons $[8,14,15]$. A crucial step for virome sequencing is the purification of VLPs from fecal samples. VLP purification is necessary for the following two reasons. First, viruses often have very small genomes compared to bacteria and host derived DNAs. Therefore, viral DNA represents a small percentage of the total DNA in a metagenomic sample [15]. This leads to a proportionally low representation of viruses in the obtained sequencing information if complete microbiomes are sequenced. Second, many phages in the intestinal microbiota are integrated into the genomes of their bacterial hosts as dormant lysogenic prophages [15]. By isolating VLPs it is possible to distinguish integrated prophage genomes from phage genomes that are associated with viral particles. While methods for VLP purification from environments such as seawater have been well-analyzed [16], methods for isolating and purifying intestinal viromes are understudied. In some environments such as the open ocean viral density is low and VLP concentrating methods such as tangential flow filtration or $\mathrm{FeCl}_{3}$ precipitation have to be used in addition to purification methods to obtain a sufficient density of VLPs for sequencing [16]. In intestinal samples, however, viruses are already highly concentrated and thus additional VLP concentration is not necessary [15].

Recent studies of intestinal viromes have used several different methods to purify VLPs from fecal samples and to prepare the DNA for sequencing $[14,15]$. While the effects of DNA amplification, library preparation and sequencing method on virus metagenomes have been investigated in great detail [17-21], a critical evaluation of methods for the purification of VLPs from intestinal content or feces has, to our knowledge, not yet been conducted.

Since phages show great variability in terms of shape, size, buoyant density, resistance to chemical and mechanical stressors, and nucleic acid content [22,23], it can be expected that the method of purification will strongly influence the degree to which specific phages and other viruses are retained in purified samples. For example, one method that has been widely used for VLP purification is cesium chloride $(\mathrm{CsCl})$ density gradient centrifugation, which purifies phages within specific density ranges and discriminates against phages that fall outside of a specified density.

The aim of our study was to evaluate methods for VLP purification from fecal samples which can be applied to samples in a reproducible and quantitative manner. These methods should be amenable to large sample numbers in parallel to enable the use of replicates. Furthermore, the methods should permit quantitative comparisons of intestinal viromes across multiple individuals and varying treatment groups. To assess the effects of purification methods on VLP recovery we used an artificial microbiota of known composition. This artificial microbiota contained six phages and two bacterial species for which complete genome sequences were available.

\section{Results and discussion}

We used an artificial intestinal microbiota sample consisting of germ-free mouse feces containing six phages (P22, T3, T7, $66, \mathrm{M} 13$ and $\Phi \mathrm{VPE} 25)$ and two bacterial strains (gram-positive: Listeria monocytogenes EGD-e and gramnegative: Bacteroides thetaiotaomicron VPI5482). Phages P22, T3, T7, and $\Phi \mathrm{VPE} 25$ represent double-stranded DNA (dsDNA) genomes, M13 has a linear single-stranded DNA (ssDNA) genome, and $\Phi 6$ has a segmented double-stranded RNA (dsRNA) genome. The phages were added in equal numbers and the total number of phage particles (plaque forming units, PFU) equaled the total number of bacteria (colony forming units, CFU) added to the sample (see Methods section for details). The two bacterial strains were added to the sample at a 1:1 ratio relative to each other.

We tested and evaluated four different methods to purify phages from mouse feces for quantitative metagenomic studies (i.e. allowing for cross-comparison of relative abundances between samples). The four methods, which we designed based on standard protocols used for virus purification [15,16,23-25], included: (i) removal of microbial cells by filtration + removal of free DNA by DNase digestion (FD), (ii) dithiothreitol treatment to degrade fecal mucus + filtration + DNase (DTT), (iii) filtration + DNase + condensation mediated phage particle precipitation with polyethylene glycol (PEG) and (iv) filtration $+\mathrm{DNase}+\mathrm{CsCl}$ density gradient centrifugation to purify phages based on density $(\mathrm{CsCl}$ ) (Figure 1, more details in methods section). A fifth treatment group consisted of the total metagenome (MG) of the original, unpurified sample. Since our study focused on the effects of phage purification methods, we used identical DNA extraction and library preparation steps for all samples and processed them in parallel.

To test the purification methods we divided the artificial microbiome sample into ten subsamples of equal mass $(0.27 \mathrm{~g}$ each). Eight subsamples were used to carry out the purification methods (FD, DTT, PEG and $\mathrm{CsCl}$ ) in duplicate. The remaining two subsamples were used for extraction of the total metagenome (MG). We will use the following abbreviations for the replicate metagenomes throughout the article: FD1 and FD2 (filtration + DNase), DTT1 and DTT2 (DTT + 


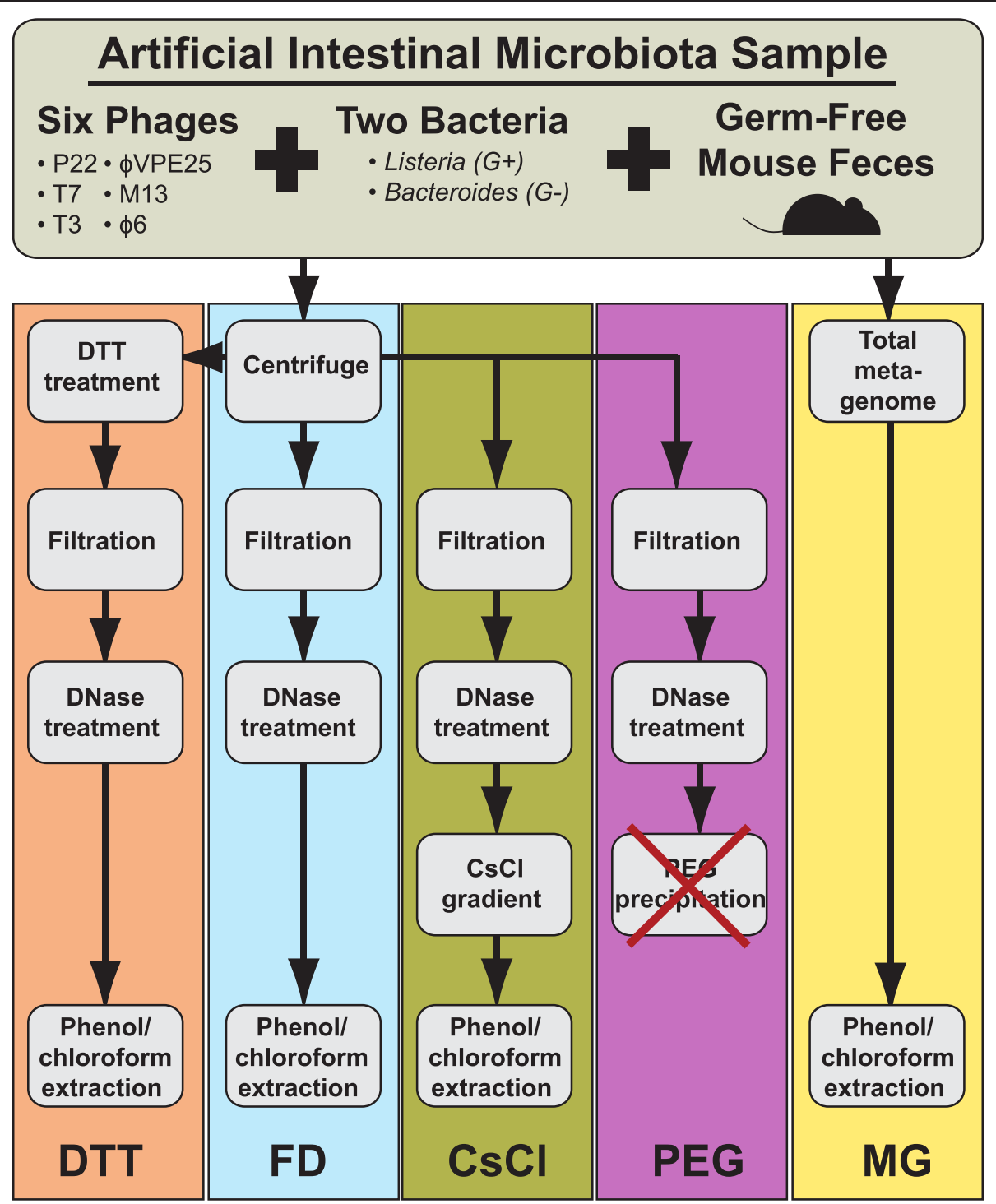

Figure 1 Schematic diagram of VLP purification methods. An artificial intestinal microbiota sample was generated by the addition of six phages and two bacterial strains to germ-free mouse feces. Upon homogenization of the mixture, the sample was split into 10 equal subsamples. Two subsamples were immediately set aside for total DNA isolation (metagenome). The remaining 8 samples were centrifuged. Upon completion of centrifugation, two samples were treated with $6.5 \mathrm{mM}$ dithiothreitol (DTT). All eight samples were filtered to remove bacteria and particulates and treated with DNase. Two samples were loaded onto a CsCl gradient and phages were banded as described in Methods. The PEG precipitation failed due to the formation of a viscous mass that impaired PEG removal by buffer exchange. After collection of the phages from the CsCl gradients, all 8 samples were extracted for total nucleic acids using phenol/chloroform extraction. G+, Gram-positive; G-, Gram-negative.

filtration + DNase), CsCl1 and $\mathrm{CsCl} 2$ (filtration + DNase + $\mathrm{CsCl}$ ), MG1 and MG2 (complete metagenome). During purification, the PEG method failed due to the formation of a viscous high molecular weight compound upon addition of the PEG to the sample filtrate. This precipitate prevented the subsequent removal of PEG by buffer exchange and these samples could no longer be processed as desired (Figure 1). In the future, the PEG method could likely be improved by removing PEG by chloroform extraction instead of buffer exchange, however, several virus groups are sensitive to chloroform and would thus be lost during PEG extraction (see e.g. [23] for a list of virus sensitivities). All eight remaining samples were subjected to paired-end sequencing on an Illumina HiSeq 2500 sequencer generating $\sim 14$ million paired-end reads per sample.

\section{DNA recovery}

All three working purification methods (FD, DTT and $\mathrm{CsCl}$ ) yielded $<10 \%$ of the DNA amount extracted from the MG samples (Table 1). MG1 yielded $636 \mathrm{ng}$ and MG2 yielded $459 \mathrm{ng}$ of DNA. The $\mathrm{CsCl}$ samples had the lowest yield, approximately $20 \mathrm{ng}$. Yields in the FD 
Table 1 Method overview/summary

\begin{tabular}{|c|c|c|c|c|}
\hline & FD & DTT & $\mathrm{CsCl}$ & MG \\
\hline Purification steps & $\begin{array}{l}\text { Filtration + } \\
\text { DNase }\end{array}$ & $\begin{array}{l}\text { DTT + Filtration + } \\
\text { DNase }\end{array}$ & $\begin{array}{l}\text { Filtration }+ \text { DNase }+\mathrm{CsCl} \\
\text { centrifugation }\end{array}$ & $\begin{array}{l}\text { Total metagenome } \\
\text { of unpurified sample }\end{array}$ \\
\hline $\begin{array}{l}\text { DNA recovered in (ng in sample 1, } \\
\text { ng in sample 2) }\end{array}$ & 42,44 & 29,34 & 21,19 & 636,459 \\
\hline Sample throughput ${ }^{a}$ & $15-20$ & $15-20$ & $6-8^{d}$ & N/A \\
\hline Total duration of protocol (days) ${ }^{b}$ & 1 & 1 & 2 & N/A \\
\hline Hands on time (hours) ${ }^{c}$ & 6 & 6 & 10 & N/A \\
\hline Special equipment needed & No & No & Ultracentrifuge & N/A \\
\hline Intra-method reproducibility & High & High & Medium & N/A \\
\hline Biases against specific phage & Weak & Weak & Strong & N/A \\
\hline Removal efficiency of mouse DNA & High & High & Very high & N/A \\
\hline $\begin{array}{l}\text { Removal efficiency of bacterial } \\
\text { DNA }\end{array}$ & Very high & Very high & Very high & N/A \\
\hline
\end{tabular}

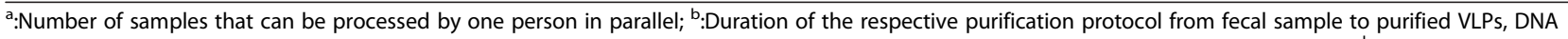
extraction time not included; ${ }^{c}$ :Hands on time needed for the number of samples that can be processed by one person in parallel (above); ${ }^{\mathrm{d}}$ :Sample number limited by rotor size of ultracentrifuge and number of density gradients that can be set up in parallel in a reasonable amount of time.

samples were around $40 \mathrm{ng}$, while the DTT samples were intermediate (DTT1: $29 \mathrm{ng}$, DTT2: $34 \mathrm{ng}$ ). Most of the reduction in DNA between the MG samples and the purified samples is likely due to the removal of bacterial and mouse DNA during sample purification.

\section{Purification efficiency, reproducibility and biases of the purification methods based on read coverage}

To evaluate the purification methods we mapped the reads from the eight metagenomes against a set of reference sequences consisting of the genomes of the input phages and bacteria plus the genomes of expected contaminants such as mouse, human and the $\Phi \mathrm{X} 174$ phage that is used as an internal control during Illumina sequencing (Figure 2, Additional file 1: Tables S1-S10). More than $97 \%$ of all reads mapped unambiguously to one of these reference genomes. The remaining reads either mapped ambiguously to several of the reference genomes or did not map to any of the reference genomes. The small number of reads that did not map to any of the reference genomes indicates that the content of unknown DNA in the samples was small.

In the following we will use differences in relative read abundances between metagenomes as an estimate of differences in relative DNA amounts in the sample. Also, for simplicity, we will refer to sequencing reads mapping to the genome of a specific organism or organism group, as $<$ organism $>$ reads e.g. mouse reads for reads mapping to the mouse genome and phage reads for the reads mapping to the genomes of all added phages (excluding the $\Phi$ X174 internal Illumina control).

All three purification methods (FD, DTT and $\mathrm{CsCl}$ ) led to an average increase of phage reads of more than 20fold. While phage reads account for $<5 \%$ of all reads in the MG samples, they account for $>80 \%$ of all reads in the purified samples (Figure 2, Additional file 1: Table S1). The highest percentage of phage reads was achieved in the $\mathrm{CsCl}$ samples, followed by the DTT samples and then the FD samples. In terms of purification efficiency the $\mathrm{CsCl}$ method is the most efficient at removing mouse DNA contamination, however, it has other drawbacks that need to be considered (see below and Table 1).

Intra-method reproducibility was observed for the FD and DTT methods, as judged by the relative read abundances of the four phages for which good read coverage was achieved (P22, $\Phi \mathrm{VPE} 25, \mathrm{~T} 3$ and T7). The intramethod ratios of relative read abundances for specific phages were between 0.92 and 1.03 , which is close to the theoretical optimum of 1 (Table 2). In contrast, much higher variability was observed between the two $\mathrm{CsCl}$ replicates, where ratios between 0.71 and 1.14 were observed (Table 2). This higher intra-method variability for the $\mathrm{CsCl}$ method may have been caused by the fraction collection, which was based on the protocol published by Thurber et al. [23]. The reproducibility might be improved by using specialized gradient-harvesting devices for gradient fractionation followed by careful evaluation of fraction densities [26,27].

As expected, variability between methods was higher than intra-method variability. The ratios of relative read abundances for specific phages between the FD and DTT methods were close to 1 indicating reproducible quantitative results between these methods. In contrast, some of the ratios between the $\mathrm{CsCl}$ method and the FD and the DTT methods deviated significantly from 1 indicating that different purification biases exist between these methods (Table 2). In particular, the read abundances of the $\Phi \mathrm{VPE} 25$ and $\mathrm{T} 7$ phages were greatly reduced in the $\mathrm{CsCl}$ purified samples (Figure 2, Additional file 1: Table S1). We also observed that when read abundances were normalized to 
A

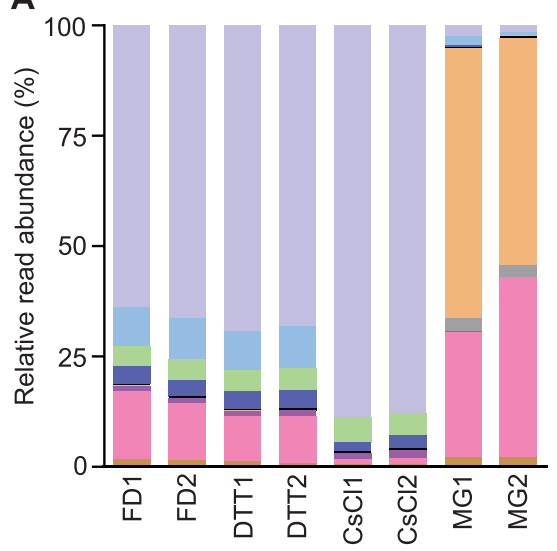

B

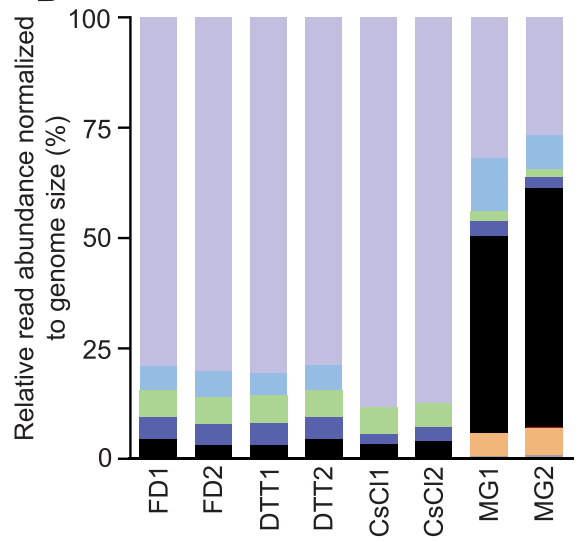

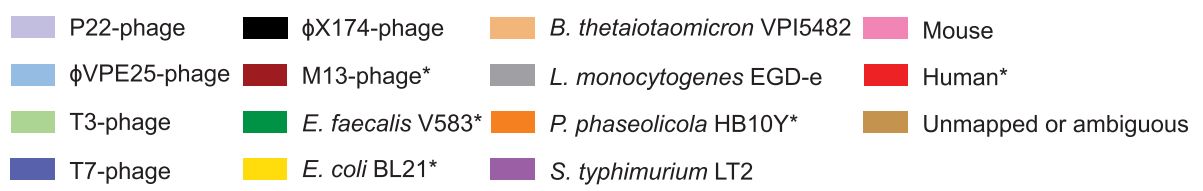

Figure 2 Read abundances from artificial intestinal microbiota samples following VLP purification or whole metagenome processing. (A) Relative read abundances (detailed data in Additional file 1: Tables S1 and S3-S10) (B) Relative read abundances after normalization to genome sizes (Detailed data in Additional file 1: Tables S2-S10). FD, Filtration + DNase - replicates 1\&2; DTT, DTT + Filtration + DNAse - replicates 1\&2; CsCl, Filtration + DNase + CsCl gradient - replicates 1\&2; MG; Total metagenome (no purification procedure) - replicates 1\&2. *Denotes organism read abundances with bars too small to be seen in the figure.

genome sizes, the ratios between the FD and DTT methods were even closer to 1 (Table 2, Additional file 1: Table S2). This can be explained by the fact that normalization of read abundance to genome size yields an estimate of relative genome copy number, which is

Table 2 Intra- and between method variation based on read abundance ratios*

\begin{tabular}{|c|c|c|c|c|c|c|c|}
\hline & \multicolumn{2}{|c|}{$\begin{array}{l}\text { Intra-method } \\
\text { variation }\end{array}$} & \multirow[b]{2}{*}{$\begin{array}{l}\mathrm{CsCl} 1 / \\
\mathrm{CsCl} 2\end{array}$} & \multirow[b]{2}{*}{$\begin{array}{l}\text { MG1/ } \\
\text { MG2 }\end{array}$} & \multicolumn{3}{|c|}{$\begin{array}{l}\text { Between } \\
\text { method variation }^{a}\end{array}$} \\
\hline & $\begin{array}{l}\text { FD1/ } \\
\text { FD2 }\end{array}$ & $\begin{array}{l}\text { DTT1/ } \\
\text { DTT2 }\end{array}$ & & & $\begin{array}{l}\mathrm{FD} / \\
\mathrm{DTT}\end{array}$ & $\begin{array}{l}\mathrm{DTT} / \\
\mathrm{CsCl}\end{array}$ & $\begin{array}{l}\mathrm{CsCl} / \\
\mathrm{FD}\end{array}$ \\
\hline \multicolumn{8}{|c|}{ Calculated with \% read abundance } \\
\hline M13 & 0.74 & 2.22 & 1.67 & 1.08 & 1.52 & 10.06 & 0.07 \\
\hline P22 & 0.96 & 1.01 & 1.01 & 1.73 & 0.95 & 0.78 & 1.36 \\
\hline фVPE25 & 0.94 & 0.92 & 0.75 & 2.33 & 0.99 & 125.23 & 0.01 \\
\hline T3 & 1.01 & 1.01 & 1.14 & 1.69 & 0.93 & 0.94 & 1.15 \\
\hline T7 & 1.03 & 1.00 & 0.71 & 1.88 & 0.94 & 1.57 & 0.68 \\
\hline \multicolumn{8}{|c|}{$\begin{array}{l}\text { Calculated with \% read abundance normalized } \\
\text { to genome size }\end{array}$} \\
\hline M13 & 0.75 & 2.24 & 1.63 & 0.73 & 1.60 & 11.20 & 0.06 \\
\hline P22 & 0.98 & 1.02 & 1.01 & 1.19 & 1.00 & 0.91 & 1.10 \\
\hline фVPE25 & 0.96 & 0.93 & 0.75 & 1.60 & 1.05 & 146.16 & 0.01 \\
\hline T3 & 1.03 & 1.01 & 1.14 & 1.16 & 0.98 & 1.09 & 0.93 \\
\hline T7 & 1.05 & 1.00 & 0.71 & 1.29 & 0.99 & 1.83 & 0.55 \\
\hline
\end{tabular}

*Perfect reproducibility would result in ratios equal to one.

${ }^{a}$ :Read abundance ratios between methods were calculated using the average read abundance in each method. less influenced by small changes in copy number of contaminating DNA originating from large genomes (e.g. mouse). Small changes in copy number of large genomes can influence read abundances significantly, because these genomes yield a read quantity proportional to their size during sequencing. Our results suggest that normalization of read abundance to genome size (if known) can improve between- sample and -method comparability.

Although all phages were mixed into the artificial microbiota sample in equal numbers (based on PFU count) their representation in the metagenomes differed greatly from an equal distribution. Since input number of phages should roughly correspond to the input genome copy number for each phage, the read abundances normalized to genome size should in theory be equal for all phages. Consequently, the ratios of these read abundances should be close to 1 within any given sample. However, even in the unaltered MG samples, this is not the case (Figure 2B, Table 3, and Additional file 1: Table S2). Both the P22 and the $\Phi \mathrm{VPE} 25$ phages have much higher read abundances in the MG samples as compared to the T3, T7 and M13 phages. For the M13 phage this distortion in read abundance can be explained by its ssDNA genome (see below). For the other phages, there are three potential explanations for why the read abundances differ from expected read abundance in the MG samples. First, phage genome copy number could be misestimated by the PFU counting method. This method only counts viable phages that are able to infect and lyse 
Table 3 Purification method biases for specific phages*

\begin{tabular}{|c|c|c|c|c|}
\hline & FD & DTT & $\mathrm{CsCl}$ & MG \\
\hline \multicolumn{5}{|c|}{ Average $\%$ read abundance normalized to genome size } \\
\hline M13 & 0.010 & 0.007 & 0.001 & 0.027 \\
\hline P22 & 79.605 & 79.695 & 87.830 & 29.338 \\
\hline фVPE25 & 5.564 & 5.310 & 0.036 & 9.749 \\
\hline T3 & 6.164 & 6.286 & 5.751 & 2.101 \\
\hline T7 & 4.879 & 4.923 & 2.691 & 2.974 \\
\hline \multicolumn{5}{|c|}{ Ratios of genome size normalized $\%$ read abundances } \\
\hline P22/ $\$$ VPE25 & 14.3 & 15.0 & 2417.5 & 3.0 \\
\hline $\mathrm{P} 22 / \mathrm{T3}$ & 12.9 & 12.7 & 15.3 & 14.0 \\
\hline $\mathrm{P} 22 / \mathrm{T7}$ & 16.3 & 16.2 & 32.6 & 9.9 \\
\hline$\phi \vee P E 25 / T 3$ & 0.9 & 0.8 & 0.0 & 4.6 \\
\hline$\phi \vee P E 25 / T 7$ & 1.1 & 1.1 & 0.0 & 3.3 \\
\hline T3/T7 & 1.3 & 1.3 & 2.1 & 0.7 \\
\hline T7/M13 & 465 & 748 & 4584 & 108 \\
\hline P22/M13 & 7587 & 12119 & 149630 & 1073 \\
\hline$\phi V P E 25 / M 13$ & 530 & 807 & 61 & 356 \\
\hline T3/M13 & 587 & 956 & 9797 & 76 \\
\hline
\end{tabular}

*In case of absence of purification biases, ratios of size normalized read abundances between phages would be identical in the unpurified control samples (MG) and the purified samples (FD, DTT and $\mathrm{CsCl}$ ).

their host. Non-viable VLPs or free phage DNA would not be considered in these measurements. It is common for phage lysates to contain a large number of nonviable VLPs, which contain nucleic acid, but are not able to produce plaques [28]. To check whether the unequal read abundances between the four dsDNA phages are caused by a misrepresentation of total VLPs by PFU counting we counted total VLPs by epifluorescence microscopy and compared them to the PFU counts (Additional file 1: Table S11). As expected the total VLP count was higher than the PFU count and the VLP/PFU ratios ranged from 4.8 to 8.2 for specific phages (Additional file 1: Table S11). However, based on this data the phage input numbers into the artificial microbiome sample are still close to the 1:1 ratio (less than two-fold difference between all phage input numbers), which we initially determined by PFU counting and thus the large observed differences in read abundances cannot be explained by differences between PFU and VLP counts. Interestingly, the P22 phage, which has the highest read abundance in all treatments, was put into the artificial microbiome sample in lowest number based on VLP counting. The unequal read abundances for phages in the MG samples could, however, be explained by free phage DNA, which would not be detected by either VLP or PFU counting. The fact that the read abundance ratios for the T7, T3 and $\Phi$ VPE25 are close to 1 in the FD and DTT samples suggests that the unequal read abundances for these phages in the MG samples were caused by free phage DNA that was removed during the purification procedure.
Second, the DNA extraction method may extract phages with different efficiencies. However, since the T3, T7 and P22 phages are structurally similar (all three are members of the Podoviridae), this seems an unlikely explanation. Third, phage genomes can carry a variety of DNA modifications [29] that could lead to biases during sequencing library preparation. It was recently shown that some DNA modifications can lead to strong exclusion biases during Illumina library preparation [30].

Differences in phage to phage ratios between the MG samples and the purified samples indicate that different methods discriminate against particular phages (Table 3, Additional file 1: Tables S2-S10). The most notable difference was a strong reduction of $\Phi \mathrm{VPE} 25$ and T7 read abundances by the $\mathrm{CsCl}$ method. There are at least three potential explanations for this reduction. First, the VPE25 and T7 particles may not have adequately accumulated in the density range extracted from the $\mathrm{CsCl}$ density gradient. Phage morphology is diverse and phages can vary widely in their buoyant densities, even when structurally related [23,31]. For example, the P22 and T7 phages both belong to the family Podoviridae, but have been shown to sediment at different density layers in a $\mathrm{CsCl}$ gradient [24]. Therefore, the bias against specific phage may have been introduced by extracting only one fraction from the $\mathrm{CsCl}$ gradient, however, the extracted density range was rather large, in theory, encompassing the densities of all phages in the sample. A second explanation for the observed reduction in read abundance in the $\mathrm{CsCl}$ samples is that some phage types degrade more rapidly in the $\mathrm{CsCl}$ gradient due to chemical or mechanical stresses. A third explanation would be that specific phage types rupture due to osmotic shock during buffer exchange releasing their genome. Osmotic shock is a common means to release nucleic acid from viruses [32]. Loss of nucleic acid during buffer exchange is unlikely, however, because the 50,000 MWCO ultrafiltration devices used are made to retain nucleic acids $>300 \mathrm{bp}$. If osmotic rupturing had occurred, the genomic DNA of the $\Phi \mathrm{VPE} 25$ and T7 bacteriophages $(>38,000 \mathrm{bp})$ would have remained in the retentate used for nucleic acid extraction. Additionally, it was previously shown for the T7 phage that it is resistant to osmotic shock induced rupture [32]. One potential way to alleviate the biases introduced by the $\mathrm{CsCl}$ method would be to extract a larger density range from the $\mathrm{CsCl}$ gradient, which may lead to better retrieval of phages, but could also diminish the "cleansing" effect of the gradient by contamination carry over. Overall the biases introduced by the FD and DTT methods are less than in the $\mathrm{CsCl}$ method.

In conclusion, the high intra-method reproducibility of the FD and DTT methods allows quantitative crosssample comparisons of phages. However, caution must be 
used when drawing conclusions about the abundances of individual phages within samples due to the fact that read abundance estimates within a sample can deviate by more than one order of magnitude from the actual input phage particle number (Table 3).

\section{Removal of bacterial and host DNA}

All three purification methods were highly successful in removing both bacterial (B. thetaiotaomicron and $L$. monocytogenes) and mouse genomic DNA as compared to the MG samples (Figure 2 and Table 4). All three purification methods led to a $>40,000$-fold enrichment of phage reads to bacterial reads (Table 4). The greatest average fold change for phage to bacterial read enrichment was observed in the $\mathrm{CsCl}$ samples (49,077-fold) and the smallest in the FD samples (41,517-fold). These fold changes indicate a removal of $>99.99 \%$ of the bacterial DNA by each purification method (Table 4). Host genomic DNA is a significant contaminant of fecal material and its presence should be considered when choosing an appropriate VLP purification method. For our study, the efficiency of removing mouse DNA from the samples differed greatly between the three purification methods. While the FD method only led to a 55fold change in the ratio of phage reads to mouse reads indicating a removal of $98.1 \%$ of mouse DNA, the $\mathrm{CsCl}$ method led to a 768-fold enrichment indicating a removal of $99.87 \%$ of mouse DNA. The observed discrepancy in removal efficiency of bacterial DNA versus

Table 4 Removal efficiency of mouse and bacterial DNA by different purification methods

\begin{tabular}{|c|c|c|c|c|}
\hline & FD & DTT & $\mathrm{CsCl}$ & MG \\
\hline \multicolumn{5}{|l|}{ Average $\%$ read abundance } \\
\hline Mouse & 14.26 & 10.49 & 1.20 & 34.63 \\
\hline Phage $^{a}$ & 82.57 & 86.79 & 96.13 & 3.62 \\
\hline Bacteria $^{\mathrm{b}}$ & 0.033 & 0.032 & 0.032 & 59.21 \\
\hline \multicolumn{5}{|c|}{ Calculations based on average $\%$ read abundance } \\
\hline Phage/mouse ratio & 5.79 & 8.27 & 80.31 & 0.10 \\
\hline $\begin{array}{l}\text { Factor of ratio change } \\
\text { compared to MG method }\end{array}$ & 55 & 79 & 768 & 1 \\
\hline $\begin{array}{l}\text { Estimated } \% \text { decrease of } \\
\text { read generating mouse } \\
\text { DNA as compared to } \mathrm{MG}^{c}\end{array}$ & 98.19 & 98.74 & 99.87 & 0.00 \\
\hline Phage/bacteria ratio & 2537.74 & 2674.62 & 2999.83 & 0.06 \\
\hline $\begin{array}{l}\text { Factor of ratio change } \\
\text { compared to MG method }\end{array}$ & 41518 & 43757 & 49078 & 1 \\
\hline $\begin{array}{l}\text { Estimated \% decrease of read } \\
\text { generating bacterial DNA as } \\
\text { compared to } \mathrm{MG}^{\mathrm{c}}\end{array}$ & 99.998 & 99.998 & 99.998 & 0.00 \\
\hline
\end{tabular}

$a^{a}$ Sum of read abundances for all added bacteriophage (this excludes the phiX174 used as Illumina internal control); ${ }^{\text {b}}$ :Sum of read abundances for the two added bacteria - L. monocytogenes and B. thetaiotaomicron; ${ }^{C}:$ Calculated based on read abundances normalized to phage read abundance. mouse DNA may be due to a larger fraction of mouse DNA existing as free DNA in mouse feces (i.e. not in nuclei or cells), which can easily pass through the filtration membrane, whereas the majority of the bacterial DNA is within cells that are efficiently removed by filtration.

An alternative explanation for the higher amount of mouse DNA in the purified samples could be the introduction of mouse DNA contamination during sample processing. Such processing-contamination has, for example, been shown to occur when using certain types of DNA purification columns [33]. However, the observed differences in mouse DNA content between purification methods strongly suggest that mouse DNA is sample derived. Assuming that free mouse DNA is responsible for the presence of mouse reads in the purified metagenomes, this would suggest that free DNA was not completely removed by DNase digestion. There are two potential reasons for this. It is possible that specific regions of the mouse genome may have been protected from DNase digestion by adhering proteins protecting the DNA from degradation or that the conditions chosen for the DNase digestion were not sufficient to achieve a complete removal of mouse DNA. To check whether specific regions of the mouse genome were protected from DNase digestion we mapped the mouse reads to the mouse genome and visualized their location using the Integrative Genomics Viewer software (Vers. 2.3.34) [34]. We found that the reads were evenly distributed along the mouse genome suggesting that incomplete digestion of mouse DNA was not due to protection of specific genomic regions.

These data suggest that efforts to remove host DNA during the purification of VLPs from fecal samples need to be intensified. This could be achieved by increasing the DNase concentration during digestion. The DNase concentration that we used in this study was $10 \mathrm{U} \mathrm{ml}^{-1}$ and corresponds to what is recommended in a standard protocol for phage purification [25]; however, it has been suggested previously that in samples from animal hosts much higher DNase concentrations may be required to remove host DNA contamination [23]. Other laboratories have used higher DNase concentrations for sequencing analyses of viruses e.g. $100 \mathrm{U} \mathrm{ml}^{-1}$ for ocean virus metagenomes [16] and $500 \mathrm{U} \mathrm{ml}^{-1}$ for PCR based analyses of viruses in serum samples [35]. We would thus recommend using higher DNase concentrations for virus purification to achieve more efficient removal of host DNA. Additionally, in our study, DNase digestions were performed at room temperature. Digestion can also be done at $37^{\circ} \mathrm{C}$ and will yield accelerated degradation and greater removal of host DNA contamination. Finally, sequencing read data should be "decontaminated" using in silico methods by mapping the reads against a host reference genome to remove sequencing reads of any remaining host DNA. 
Phage genome recovery by genome assembly

To determine if whole phage genomes could be retrieved from the Illumina 100 bp paired-end read data, we performed de novo assemblies from all datasets (Table 5). We retrieved complete or almost complete genomes for four phages from all datasets (P22, $\Phi \mathrm{VPE} 25, \mathrm{~T} 3$ and T7, Table 5), despite the fact that we did not do any assembly procedure optimization (i.e. trying different parameters, binning reads or using assemblers optimized for metagenomes). Our assemblies even contained contigs of the ssDNA M13 phage for which only very few reads were sequenced. The largest M13 contig ( 1 kbp) was assembled from the FD1 sample representing a significant portion of the $6.4 \mathrm{kbp}$ genome of the M13 phage.

\section{Effects of nucleic acid composition of phage genomes}

In our study we focused on the recovery of dsDNA phages. Nevertheless, we added two phages with nondsDNA genomes ( $\Phi 6$ and M13) to the artificial microbiota sample to see if these phages could be recovered by any of the tested methods. To determine the recovery of the $\Phi 6$ phage, which has a dsRNA genome, we did a cDNA synthesis using the FD and MG samples. The recovered $\Phi 6$ RNA in these samples was too low and the sequencing library preparation failed. Consequently not a single read was retrieved for $\$ 6$ in any of the purified samples or in the MG samples. Surprisingly, for the M13 phage, which has a ssDNA genome, a small number of reads were sequenced in all purified samples and the MG samples. In theory sequencing of ssDNA should be prevented by the Illumina library preparation protocol which requires dsDNA as input [36]. T4 DNA ligase, commonly used for Illumina adapter ligation, works preferentially with dsDNA and excludes ssDNA. However, it has been shown that the T4 DNA ligase can ligate ssDNA albeit with a very low efficiency [37], which might explain why small amounts of M13 ssDNA were sequenced. This suggests that the tested methods are suitable for qualitatively assessing ssDNA viruses, if a large enough number of sequencing reads is generated. However, to get a clearer picture of RNA and ssDNA viruses additional steps should be added to these protocols.

Several approaches could be used to achieve greater sequencing coverage of ssDNA viruses. First, multiple displacement amplification (MDA), which is known to preferentially amplify ssDNA virusus, can be used to generate dsDNA [23,38-40]. MDA, however, has the caveat of introducing strong biases and the resulting sequences can only be used for a qualitative assessment of virus diversity and not quantitative analyses [19-21]. Second, random hexamer primers and DNA polymerase I can be used to convert ssDNA to dsDNA [41]. Third, ssDNA ligase can be used to ligate Illumina adapters directly to ssDNA during library preparation, however, this method has not been tested on viral DNA [30]. For the analysis of RNA viruses, RNA can be amplified and converted to dsDNA by reverse transcriptase $[23,41]$. However, care must be taken to avoid RNA degradation by nucleases during sample preparation. Furthermore, nucleic acid types can be separated using hydroxyapatite chromatography prior to dsDNA generation [41].

Table 5 Assembly statistics

\begin{tabular}{|c|c|c|c|c|c|c|c|c|c|}
\hline & Published sequence & FD1 & FD2 & DTT1 & DTT2 & $\mathrm{CsCl} 1$ & $\mathrm{CsCl} 2$ & MG1 & MG2 \\
\hline P22 No. of contigs & 1 & 1 & 1 & 1 & 1 & 1 & 1 & 1 & 1 \\
\hline P22 largest contig (bp) ${ }^{a}$ & 41660 & 41737 & 41737 & 41737 & 41737 & 41737 & 41659 & 41737 & 41737 \\
\hline P22 coverage $(x$ fold $)$ & $\mathrm{N} / \mathrm{A}$ & 3651 & 3138 & 3925 & 4027 & 5489 & 4998 & 146 & 107 \\
\hline T7 No. of contigs & 1 & 1 & 2 & 2 & 1 & 1 & 1 & 4 & 1 \\
\hline T7 largest contig (bp) & 39937 & 39855 & 35301 & 35209 & 39344 & 39855 & 39855 & 35472 & 39855 \\
\hline T7 coverage $(x$ fold $)$ & $\mathrm{N} / \mathrm{A}$ & 256 & 220 & 257 & 273 & 168 & 202 & 16 & 11 \\
\hline T3 No. of contigs & 1 & 1 & 4 & 4 & 2 & 1 & 1 & 2 & 1 \\
\hline T3 largest contig (bp) & 38209 & 37540 & 33483 & 33392 & 37540 & 36121 & 37541 & 33656 & 36120 \\
\hline T3 coverage $(x$ fold $)$ & $\mathrm{N} / \mathrm{A}$ & 293 & 265 & 305 & 321 & 381 & 310 & 10 & 8 \\
\hline фVPE25 No. of contigs & 1 & 1 & 1 & 1 & 1 & 1 & 1 & 1 & 2 \\
\hline фVPE25 largest contig (bp) & 86524 & 86520 & 86522 & 86518 & 86542 & 86496 & 86505 & 86534 & 86540 \\
\hline фVPE25 coverage $(x$ fold $)$ & $\mathrm{N} / \mathrm{A}$ & 256 & 225 & 253 & 284 & 1.9 & 2.3 & 55 & 30 \\
\hline M13 No. of contigs & 1 & 7 & 4 & 8 & 4 & 1 & 0 & 1 & 0 \\
\hline M13 largest contig (bp) & 6407 & 1085 & 900 & 814 & 586 & 230 & 0 & 268 & 0 \\
\hline M13 coverage ( $x$ fold) & N/A & 0.35 & 0.55 & 0.43 & 0.39 & 0.18 & 0 & 0.14 & 0 \\
\hline
\end{tabular}

${ }^{\mathrm{a}}$ :Assemblies of the P22 genome from our datasets are slightly larger than the reference genome, which is likely due to the quasi-circular nature of the P22 genome, which makes it impossible for the assembly algorithm to determine the exact start and end of the genome. 


\section{Conclusions}

In this study we used artificial microbiota samples, with known bacterial and viral composition, to evaluate four methods for the purification of VLPs from feces for quantitative comparisons of intestinal viromes. One method (PEG) failed during the purification procedure. All other methods succeeded in isolating VLPs and are suitable for quantitative sample comparison if one considers their limitations and pitfalls (discussed above). There are some notable differences in the ease of use, throughput, and performance of the different methods (Table 1). These differences should be considered when choosing the appropriate method. While the $\mathrm{CsCl}$ method outperformed the FD and DTT methods in removal efficiency of host derived DNA, the FD and DTT methods showed a lower discrimination against specific phage species and also yielded more total DNA. An additional consideration during study design should be the number of samples that will be analyzed. The FD and DTT methods allow for a much higher sample throughput, because they do not include the time consuming $\mathrm{CsCl}$ density gradient centrifugation step, which also limits the number of samples that can be processed in parallel (Table 1). As discussed above, the lower efficiency of host DNA removal in the FD and DTT methods may be alleviated by increasing DNase concentrations during DNase digestion. In this study we only observed small differences in the performance of the FD and DTT methods. Therefore, the DTT method can be applied to the processing of large scale fecal samples when mucus degradation is required to prevent filter clogging during VLP purification. A crucial additional consideration for the design of virome studies is the inclusion of appropriate negative controls to be carried from sample purification to metagenome sequencing and in silico analyses [42].

\section{Methods \\ Mice}

Germ-free C57BL/6 J mice were bred and reared in sterile isolators [43] at the UT-Southwestern Medical Center's animal barrier facility. Animal protocols were approved by the Institutional Animal Care and Use Committees of UTSouthwestern.

\section{Bacteria, bacteriophages and culturing conditions}

A total of six phages were used in this study. Three phages belonging to the Family Podoviridae, with linear dsDNA genomes, were purchased from the American Type Culture Collection (ATCC ${ }^{\oplus}$, Manassas, VA). These phages included P22 (ATCC $19585-$ B1 $^{\mathrm{m}}{ }^{\circ}$ ), T7 (ATCC ${ }^{\bullet}$ BAA-1025-B2 ${ }^{\mathrm{mm}}$ ), and T3 (ATCC BAA-1025-B1 ${ }^{1 \mathrm{~m}}$ ), the latter was originally sold to us as T4 $\left(\mathrm{ATCC}^{\circ} 11303-\mathrm{B}^{\mathrm{mm}}\right)$. During the course of our study we determined that the T4 phage stock prepared by ATCC $^{\circ}$ was actually the T3 type strain. Phage $\Phi$ VPE25 was isolated from a municipal waste water source and the ФVPE25 genome has been sequenced (manuscript in preparation). $\Phi \mathrm{VPE} 25$ is a member of the Siphoviridae family of dsDNA viruses and infects Enterococcus faecalis (data not shown). In addition to dsDNA phages we used phages M13 (New England Biolabs, Ipswich, MA) which belongs to the family Inoviridae and contains a ssDNA genome and $\phi 6$ (a gift from P. Turner) a member of the Cystoviridae family harboring a segmented dsRNA genome [44].

All bacterial hosts were grown aerobically at $37^{\circ} \mathrm{C}$ except for Pseudomonas syringae pathovar phaseolicola $\mathrm{HB} 10 \mathrm{Y}$ which was grown aerobically at $25^{\circ} \mathrm{C}$ [44]. Escherichia coli B (ATCC ${ }^{\circ} 11303^{\mathrm{rm}}$ ) and Salmonella enterica subsp. enterica serovar typhimurium LT2 (ATCC ${ }^{\circ} 19585^{\mathrm{mm}}$ ) were grown in ATCC $^{\circ} 129$ medium (per liter; 3 g Beef Extract, 5 g Peptone, and $5 \mathrm{~g} \mathrm{NaCl}$ ), E. faecalis V583 was grown in Bacto ${ }^{\circ}$ Brain Heart Infusion medium (BHI, Becton Dickinson, Franklin Lakes, NJ) [45], and P. phaseolicola was grown in $\mathrm{LC}$ medium (per liter; $10 \mathrm{~g}$ tryptone, $5 \mathrm{~g}$ yeast extract, and $10 \mathrm{~g} \mathrm{NaCl}$ per liter, $\mathrm{pH}$ 7.5). For agar overlays during phage propagation, bacterial hosts were grown on their respective medium containing $1.5 \%$ base agar and $0.7 \%$ top agar. The bacteria used for the artificial intestinal microbiota were grown as follows; Listeria monocytogenes EGD-e [46] was grown aerobically on BHI and Bacteroides thetaiotaomicron VPI5482 [47] was grown anaerobically using the GasPak ${ }^{\text {mix }}$ EZ Container System (Becton Dickinson) on TYG medium (per liter; $10 \mathrm{~g}$ tryptone, $5 \mathrm{~g}$ yeast extract, $2 \mathrm{~g}$ D-glucose, $0.5 \mathrm{~g}$ cysteine, $13.6 \mathrm{~g}$ $\mathrm{KH}_{2} \mathrm{PO}_{4}, 17.4 \mathrm{~g} \mathrm{~K}_{2} \mathrm{HPO}_{4}, 0.25 \mathrm{~g} \mathrm{NaHCO}_{3}, 0.4 \mathrm{~g} \mathrm{FeSO}_{4}$, $80 \mathrm{mg} \mathrm{NaCl}, 20 \mathrm{mg} \mathrm{MgSO}{ }_{4} \cdot 7 \mathrm{H}_{2} \mathrm{O}, 8 \mathrm{mg} \mathrm{CaCl} \cdot 2 \mathrm{H}_{2} \mathrm{O}$, $1 \mathrm{~g}$ Vitamin-K, $0.25 \mathrm{~g}$ resazurin, $0.24 \mathrm{mg}$ hematin [prepared by dissolving $12 \mathrm{mg}$ of hematin in $10 \mathrm{ml}$ of $0.2 \mathrm{M}$ histidine, $\mathrm{pH}$ 8.0]).

\section{Phage propagation}

P22, T7, and T3: Propagation and purification of the enteric phages $\mathrm{P} 22, \mathrm{~T} 7$, and T3 were similar. A single colony of E. coli B (T7 and T3) or S. typhimurium LT2 (P22) was inoculated into $5 \mathrm{ml}$ of ATCC 129 medium and grown overnight at $37^{\circ} \mathrm{C}$ with shaking (250 rpm). The bacteria were subcultured into $300 \mathrm{ml}$ of fresh $\mathrm{ATCC}^{\bullet} 129$ medium to an $\mathrm{OD}_{600}$ of 0.015 . The cultures were grown for $2.5 \mathrm{hrs}$ at $37^{\circ} \mathrm{C}$ with shaking. Lyophilized phages provided by ATCC $^{\ominus}$ were reconstituted in $1 \mathrm{ml}$ of ATCC $^{\bullet} 129$ medium and $30 \mu \mathrm{l}$ of each phage solution was added to its respective host strain's culture. These cultures were incubated for 3 hours (T7 and T3) and 3.5 hours $(\mathrm{P} 22)$ at $37^{\circ} \mathrm{C}$ with shaking to achieve host lysis. The cultures were transferred to $500 \mathrm{ml}$ centrifuge bottles and spun at $2820 \times \mathrm{g}$ for $20 \mathrm{~min}$ at $4^{\circ} \mathrm{C}$. Any remaining bacterial pellet was discarded and the culture supernatants were 
filtered using a $0.22 \mu \mathrm{m}$ bottle top filter (Corning, Tewksbury, MA). The clarified culture supernatants were treated with $10 \mathrm{U} \mathrm{ml}^{-1}$ each of RNase and DNase (Sigma-Aldrich, St. Louis, MO) for $1 \mathrm{hr}$ at room temperature. $1 \mathrm{M}$ solid $\mathrm{NaCl}$ and 10\% (w/v) PEG 8000 was then added to the culture fluid and incubated on ice for four hours. The precipitated phage particles were transferred to a clean $500 \mathrm{ml}$ centrifuge bottle and spun at $8000 \times \mathrm{g}$ for $20 \mathrm{~min}$ at $4^{\circ} \mathrm{C}$. The phage pellets were resuspended in $3 \mathrm{ml}$ of SM-plus buffer $(100 \mathrm{mM}$ $\mathrm{NaCl}, 50 \mathrm{mM}$ Tris $\cdot \mathrm{HCl}, 8 \mathrm{mM} \mathrm{MgSO}{ }_{4} \cdot 7 \mathrm{H}_{2} \mathrm{O}, 5 \mathrm{mM}$ $\mathrm{CaCl}_{2} \cdot 2 \mathrm{H}_{2} \mathrm{O}, \mathrm{pH}$ 7.4). The resuspended phages were layered on top of $\mathrm{CsCl}$ step gradients consisting of increasing $\mathrm{CsCl}$ densities of $1.7 \mathrm{~g} / \mathrm{ml}, 1.6 \mathrm{~g} / \mathrm{ml}$, and $1.42 \mathrm{~g} / \mathrm{ml}(\mathrm{P} 22$ and T3) and $1.7 \mathrm{~g} / \mathrm{ml}, 1.6 \mathrm{~g} / \mathrm{ml}$, and $1.45 \mathrm{~g} / \mathrm{ml}$ (T7) in Ultra-Clear ${ }^{\mathrm{Tm}} 14 \times 89 \mathrm{~mm}$ centrifuge tubes (Beckman Coulter, Indianapolis, IN), followed by spinning in a Beckman Coulter XE-90 Ultracentrifuge at $66,000 \times \mathrm{g}$ for 16 hours at $4^{\circ} \mathrm{C}$, using a SW Ti-41 swinging bucket rotor. After ultracentrifugation phages were observed as visible hazy blue/white bands at the top of the $1.6 \mathrm{~g} / \mathrm{ml} \mathrm{CsCl}$ density zone. The phages within the condensed bands $(0.5-1 \mathrm{ml})$ were removed from the gradient tubes using a syringe fitted with a $23 \mathrm{G}$ needle and added to $5 \mathrm{ml}$ of fresh SM-plus buffer. The samples were transferred to Amicon ${ }^{\circ}$ Ultra Centrifugal Filters, 50,000 MWCO (EMD Millipore, Billerica, MA) and spun at $3220 \times \mathrm{g}$ for $5 \mathrm{~min}$, resuspended in $4 \mathrm{ml}$ of SM-plus buffer and centrifuged again. This step was repeated at least 3 times to remove the majority of the $\mathrm{CsCl}$. After buffer exchange the final retentate was added to $2 \mathrm{ml}$ of SM-plus buffer, filtered with a $0.22 \mu \mathrm{m}$ SFCA syringe filter (Thermo ScientificNalgene, Waltham, MA), and stored at $4^{\circ} \mathrm{C}$.

Ф6: For the amplification and purification of phage $\Phi 6$ a method similar to Turner et al. was followed [44]. Briefly, a single colony of $P$. phaseolicola $\mathrm{HB} 10 \mathrm{Y}$ was grown overnight in $5 \mathrm{ml}$ of LC medium. $200 \mu \mathrm{l}$ of the overnight $P$. phaseolicola culture was added to each of 6 sterile $14 \mathrm{ml}$ round-bottom Falcon tubes (Corning). To each tube $4.3 \times 10^{3}$ PFU of $\phi 6$ was added and then mixed with $3 \mathrm{ml}$ of molten LC top agar and poured onto the surface of an LC agar plate. The plates were incubated at room temperature overnight. $3 \mathrm{ml}$ of LC broth was added to each plate and the top agar was collected. This mixture was centrifuged at 36,000 $\times \mathrm{g}$ for $30 \mathrm{~min}$ at $4^{\circ} \mathrm{C}$ in an FX6100 fixed angle rotor (Beckman Coulter). The supernatant was transferred to a $25 \times 89 \mathrm{~mm}$ polycarbonate cap assembly centrifuge tube (Beckman Coulter) and centrifuged at 73,000 $\times \mathrm{g}$ for 2 hours at $4^{\circ} \mathrm{C}$ in a Ti 70 fixed angle rotor to pellet the phages. The phage pellet was resuspended in $1 \mathrm{ml}$ of Buffer A (per liter; $1.9 \mathrm{~g} \mathrm{KH}_{2} \mathrm{PO}_{4} \cdot 3 \mathrm{H}_{2} \mathrm{O}, 0.25 \mathrm{~g}$ $\mathrm{MgSO}_{4} \cdot 7 \mathrm{H}_{2} \mathrm{O}, \mathrm{pH}$ 7.5). A sucrose density gradient was poured in an Ultra-Clear ${ }^{\mathrm{rm}} 14 \times 89 \mathrm{~mm}$ centrifuge tube. The gradient steps consisted of $30 \%, 25 \%, 20 \%, 15 \%$, and $10 \%$ sucrose dissolved in Buffer A. The suspended phages were layered on top of the $10 \%$ sucrose step and centrifuged in a SW Ti-41 rotor at $66,000 \times \mathrm{g}$ for $1 \mathrm{hr}$ at $15^{\circ} \mathrm{C}$. The phage band was removed from the gradient ( $\sim 1 \mathrm{ml})$ using a syringe and $23 \mathrm{G}$ needle and suspended in Buffer A. The sample was transferred to a $25 \times 89 \mathrm{~mm}$ polycarbonate cap assembly centrifuge tube and centrifuged at 73,000 $\times \mathrm{g}$ for $2 \mathrm{hrs}$. The resulting supernatant was saved and the phage pellet resuspended in $0.6 \mathrm{ml}$ of Buffer A. The supernatant was centrifuged a second time to collect any remaining phages for 4 hrs at 73,000 $\times$ g. Again the supernatant was saved and the pellet was suspended in $0.6 \mathrm{ml}$ of Buffer A. It was determined by plaque assay that the recovered phages from the pellet were of insufficient titer and upon analysis of the supernatants, a large proportion of the phages did not pellet by ultracentrifugation. Therefore, the pelleted phage samples and the decanted supernatants were pooled and transferred to an Amicon Ultra Centrifugal Filter, 10,000 MWCO (EMD Millipore) and spun at $3220 \times \mathrm{g}$ for $5 \mathrm{~min}$. The filtration unit was filled with fresh Buffer A and spun at $3220 \times \mathrm{g}$ for $7 \mathrm{~min}$. This was repeated a second time resulting in a final retentate volume of $1.5 \mathrm{ml}$ which was stored at $4^{\circ} \mathrm{C}$. ФVPE25: A $5 \mathrm{ml}$ culture of BHI was inoculated with a single colony of E. faecalis V583 and grown overnight. The next day $300 \mathrm{ml}$ of BHI medium was inoculated with the overnight culture to an $\mathrm{OD}_{600}$ of 0.025 . The culture was transferred to a $37^{\circ} \mathrm{C}$ shaking incubator and grown until the $\mathrm{OD}_{600}$ reached 0.7. The culture was removed from the incubator and $10 \mathrm{mM} \mathrm{MgSO}_{4}$. $7 \mathrm{H}_{2} \mathrm{O}$ was added followed by the addition of $\Phi \mathrm{VPE} 25$ particles. The culture was incubated at room temperature for $10 \mathrm{~min}$ and then placed in the $37^{\circ} \mathrm{C}$ shaking incubator for 4 hours. The culture was transferred to a $500 \mathrm{ml}$ centrifuge bottle and spun at $2820 \times \mathrm{g}$ for $20 \mathrm{~min}$ at $4^{\circ} \mathrm{C}$. The supernatant was collected and filtered through a $0.45 \mu \mathrm{m}$ bottle top filter (Thermo Scientific-Nalgene). The filtered culture fluid was treated with $10 \mathrm{U} \mathrm{ml}^{-1}$ of both RNase and DNase for $1 \mathrm{hr}$ at room temperature. $1 \mathrm{M}$ solid $\mathrm{NaCl}$ and 10\% (w/v) polyethylene glycol (PEG) 8000 was dissolved into the culture fluid and incubated on ice overnight at $4^{\circ} \mathrm{C}$. The phages were pelleted by centrifugation at $8000 \times \mathrm{g}$ for $20 \mathrm{~min}$ at $4^{\circ} \mathrm{C}$. The phage pellet was resuspended in $3 \mathrm{ml}$ of SM-plus buffer and extracted with $1 / 5^{\text {th }}$ volume of chloroform and centrifuged at $16,000 \times \mathrm{g}$ for $2 \mathrm{~min}$. The aqueous phase containing the phages was collected, brought up to $4.5 \mathrm{ml}$ with SM-plus buffer. $2.2 \mathrm{~g}$ of $\mathrm{CsCl}$ was dissolved into the sample, which was placed on top of the $1.45 \mathrm{~g} / \mathrm{ml} \mathrm{CsCl}$ density layer of a $\mathrm{CsCl}$ gradient consisting of $1.7 \mathrm{~g} / \mathrm{ml}, 1.5 \mathrm{~g} / \mathrm{ml}$, and $1.45 \mathrm{~g} / \mathrm{ml}$ 
steps. This was placed in a SW Ti-41 rotor and spun at $59,764 \times \mathrm{g}$ for $2 \mathrm{hrs}$ at $4^{\circ} \mathrm{C}$. The phage band $(\sim 1-2 \mathrm{ml})$ was removed using a syringe and $23 \mathrm{G}$ needle and transferred to Slide-A-Lyzer ${ }^{\circ}$ dialysis cassette, 10,000 MWCO, (Thermo Scientific) and dialyzed twice against $2 \mathrm{~L}$ of SMplus buffer to remove the $\mathrm{CsCl}$. After dialysis the phage sample was removed from the cassette and stored at $4^{\circ} \mathrm{C}$.

Phage titers were determined with the standard softagar overlay method using the phage specific host strains and media $[25,48]$.

\section{Quantification of phage particles by fluorescence microscopy}

To determine the absolute quantities of dsDNA phages P22, T3, T7, and $\Phi$ VPE25 in stock solutions, a method similar to that described by Thurber et al. was used [23]. Concentrated phage stocks were diluted 10,000X-100,000X in SM-plus buffer. An aliquot of diluted phage was added to $5 \mathrm{ml}$ of SM-plus and applied to the column of a $25-\mathrm{mm}$ microanalysis filter holder with a fritted glass support (VWR International, Radnor, PA) holding a 25-mm $0.02 \mu \mathrm{m}$ aluminum oxide Annodisc filter (GE Healthcare Bio-Sciences, Pittsburgh, PA). Vacuum was applied $(<10$ p.s.i.) until all of the fluid passed through the filter. Filtration was performed in triplicate. After filtration the filter was placed (sample side up) onto a $120 \mu \mathrm{l}$ drop of $5 \mathrm{X} \mathrm{SYBR}^{\circ}$ Gold in a sterile petri dish and incubated at room temperature for 30 minutes in the dark. The filter was washed once by placing onto a $120 \mu \mathrm{l}$ drop of SM-plus for 30 seconds and excess liquid was wicked away using a Kimwipe. Filters were mounted onto glass microscope slides with cover slips using Tris-buffered Fluoro-Gel (Electron Microscopy Sciences, Hatfield, PA) and phage particles were imaged on an Axio Imager.M1 fluorescence microscope (Carl Zeiss Microscopy, Göttingen, Germany) coupled to an XCite Series 120 illumination lamp (EXFO Life Sciences, Montreal, Quebec, Canada) at 1000X magnification. Fluorescent phage particles were counted from a total of 5 random fields per filter and averaged. Absolute phage numbers were calculated based on the average phage counts per field taking into account the area of one field $\left(0.00596 \mathrm{~mm}^{2}\right)$, the area of the Annodisc filter $\left(490.874 \mathrm{~mm}^{2}\right)$, and back calculating based on the dilution factor.

\section{Generation of the artificial microbiota master mix sample} One artificial gut microbiome sample master mix (for a total of 10 samples) was produced by the introduction of various pure cultures of bacteria and phages into feces from germ-free C57BL/6 J mice. $2.5 \times 10^{9} \mathrm{CFU}$ each of $L$. monocytogenes and $B$. thetaiotaomicron were added to $2.7 \mathrm{~g}$ of freshly collected germ-free mouse feces $(0.27 \mathrm{~g}$ feces per sample). Then, for each of the six phages - P22,
T7, T3, $\Phi$ VPE25, M13, and $\phi 6-8.3 \times 10^{8}$ PFU each were added to the sample resulting in a total of $5 \times 10^{9}$ phage particles in the master mix. The number of phage particles to be used in the master mix was determined in a preexperiment by quantifying the amount of DNA in $10^{10}$ PFU of $\Phi$ VPE25, which was 2,355 ng, and the amount of DNA that could be extracted from VLPs isolated by $\mathrm{CsCl}$ density gradient centrifugation from $0.6 \mathrm{~g}$ of conventional mouse feces, which was $12 \mathrm{ng}$. Based on this we estimated that $\sim 5 \times 10^{7}$ VLPs could be isolated from $0.6 \mathrm{~g}$ of mouse feces. To account for VLP loss during purification procedures we added 10-fold excess PFU of each phage for each of the 10 individual samples in the master mix. After addition of the bacteria and phages to the feces master mix, $12 \mathrm{ml}$ of SM-plus buffer was added and the master mix was homogenized by rotor and stator (Omni International, Kennesaw, GA). The master mix was then weighed and aliquoted into 10 equal samples.

\section{Purification procedures}

Immediately upon allocation of the 10 individual samples, two samples were extracted for nucleic acid (see below). These samples denote the Complete Metagenome (MG). The remaining 8 samples were centrifuged at $2500 \times \mathrm{g}$ for $5 \mathrm{~min}$, the supernatant was collected and then centrifuged a second time at $5000 \times \mathrm{g}$ for $15 \mathrm{~min}$. At this point various modifications to the procedure were preformed to enrich for virus-like particles (VLPs) by different means: Purification by filtration (FD) - Two samples were filtered through a $0.45 \mu \mathrm{m}$ Millex-HV low protein binding PVDF syringe filter (EMD Millipore) which was washed with $500 \mu \mathrm{l}$ of SM-plus buffer. The filtered fluid was treated with $10 \mathrm{U} \mathrm{ml}^{-1}$ of DNase at room temperature for 1 hour and then extracted for nucleic acids. Mucolytic agent and filter purification (DTT) - One problem that occurs when using large quantities of intestinal contents for phage isolation is interference of intestinal mucus during filtration. A procedure that can be used to reduce the viscosity of mucous containing samples during phage preparation, prior to filtration, is treatment with the reducing reagent dithiothreitol (DTT) [13]. However, the effects of DTT on phage particle recovery during purification are not well understood. Therefore, two samples were first treated with $6.5 \mathrm{mM} \mathrm{DTT}$ for $1 \mathrm{hr}$ at $37^{\circ} \mathrm{C}$, filtered through a $0.45 \mu \mathrm{m}$ PVDF syringe filter which was washed with $500 \mu \mathrm{l}$ of SM-plus buffer, treated with $10 \mathrm{U}$ $\mathrm{ml}^{-1}$ of DNase at room temperature for 1 hour, and the nucleic acid extracted. Purification by filtration and cesium chloride density gradient centrifugation ( $\mathrm{CsCl}$ - A common procedure for the purification of phages from complex intestinal contents is to purify the particles by $\mathrm{CsCl}$ centrifugation $[7,8,23]$. Two samples were filtered through a $0.45 \mu \mathrm{m}$ syringe filter, washed with $500 \mu \mathrm{l}$ of SM-plus buffer, and treated with $10 \mathrm{U} \mathrm{ml}^{-1}$ of DNase at 
room temperature for 1 hour. After DNase treatment the samples were loaded onto a $\mathrm{CsCl}$ gradient composed of $1.7 \mathrm{~g} / \mathrm{ml}, 1.5 \mathrm{~g} / \mathrm{ml}, 1.35 \mathrm{~g} / \mathrm{ml}$ steps and centrifuged for 16 hours in a SW Ti-41 rotor at $4^{\circ} \mathrm{C}$. The interface between the 1.35 and $1.5 \mathrm{~g} / \mathrm{ml}$ density region $(\sim 1 \mathrm{ml})$ was collected and the $\mathrm{CsCl}$ was removed by three $4 \mathrm{ml}$ volume SM-plus buffer exchanges in an Amicon ${ }^{\circ}$ Ultra Centrifugal Filter (50,000 MWCO). The samples were then extracted for total DNA. Purification by filtration and precipitation (PEG) - The final method was based on a phage precipitation technique where PEG8000 is added to sequester water molecules forcing the phages to aggregate. The precipitated phages can then be collected by centrifugation $[24,25]$. Two samples were filtered through a $0.45 \mu \mathrm{m}$ PVDF syringe filter which was washed with $500 \mu$ of SMplus buffer, treated with $10 \mathrm{U} \mathrm{ml}^{-1}$ of DNase at room temperature for 1 hour. To each sample $1 \mathrm{M} \mathrm{NaCl}$ and $10 \%(\mathrm{w} / \mathrm{v})$ PEG8000 was added, the phages were allowed to precipitate on ice for 2 hours. The precipitate was collected by centrifugation at $8000 \times \mathrm{g}$ for 20 minutes and resuspended in $1 \mathrm{ml}$ of SM-plus buffer. To remove the PEG8000 prior to nucleic acid extraction a buffer exchange was attempted by the addition of $4 \mathrm{ml}$ of SM-plus to the sample and centrifugation in a Amicon ${ }^{\circ}$ Ultra Centrifugal Filter (50,000 MWCO). However, the PEG precipitation resulted in an extremely viscous solution that could not pass through the centrifugal filter during buffer exchange. Therefore, this sample was omitted from further analysis.

An important consideration for the purification method design is the choice of pore size to be used for filtration steps. The right balance between removing the unwanted microbial cells and letting viruses pass has to be found. Commonly used pore sizes for virus isolation are 0.2 and $0.45 \mu \mathrm{m}[16,49-51]$. We chose a $0.45 \mu \mathrm{m}$ pore size for the tested purification methods because several bacteriophage groups from the mammalian intestine have members that are larger than $0.2 \mu \mathrm{m}$ (e.g. Myoviridae and Siphoviridae [52]). Additionally, giant eukaryotic viruses $(\sim 400 \mathrm{~nm})$ were recently discovered in human intestinal content [50]. Since, most microbial cells in the mammalian intestine are larger than $0.45 \mu \mathrm{m}$ [53], use of $0.45 \mu \mathrm{m}$ filters should be possible. In contrast, some environments harbor abundant bacteria smaller than $0.45 \mu \mathrm{m}$, for example Pelagibacter ubique in the open ocean [54], where the use of a $0.2 \mu \mathrm{m}$ pore size for virus isolation may be crucial.

\section{Nucleic acid extraction}

For all samples, total nucleic acids were extracted using the following protocol. $50 \mu \mathrm{g} / \mathrm{ml}$ Proteinase- $\mathrm{K}$ and $0.5 \%$ sodium dodecyl sulfate (SDS) was added to each sample and incubated at $56^{\circ} \mathrm{C}$ for 1 hour. Samples were mixed with an equal volume of phenol/chloroform/isoamyl alcohol and vigorously extracted by shaking for $10 \mathrm{sec}$. The samples were centrifuged at $16,000 \times \mathrm{g}$ for $2 \mathrm{~min}$.
The aqueous phase was transferred to a clean microfuge tube and extracted with an equal volume of chloroform. The samples were centrifuged at $16,000 \times \mathrm{g}$ for $2 \mathrm{~min}$ and the chloroform extraction was repeated. The aqueous phase was transferred to a clean microfuge tube and the nucleic acids precipitated by the addition of $0.3 \mathrm{M}$ sodium acetate $(\mathrm{pH}, 7.0)$ and 2.5 volumes of isopropanol. The samples were incubated at $-20^{\circ} \mathrm{C}$ for 2 hours and then centrifuged at $16,000 \times \mathrm{g}$ for 30 minutes. The precipitated nucleic acid was washed once with $700 \mu \mathrm{l}$ of $70 \%$ ethanol and centrifuged at $16,000 \times \mathrm{g}$ for $10 \mathrm{mi}-$ nutes. The supernatant was decanted and the pellets were allowed to dry at room temperature for $10 \mathrm{~min}$. The nucleic acid was resuspended in EB buffer $(10 \mathrm{mM}$ Tris $\cdot \mathrm{HCl}, \mathrm{pH}$ 8.5) and further purified using the MinElute ${ }^{\bullet}$ Reaction Cleanup Kit (Qiagen, Valencia, CA).

\section{CDNA synthesis}

For the preparation of cDNA from total RNA, nucleic acid extracted metagenome samples were treated with $10 \mathrm{U} \mathrm{ml}^{-1}$ of DNase for 1 hour at room temperature. RNA was purified using an RNeasy Mini Kit (Qiagen). RNA was eluted from the RNeasy columns in $30 \mu \mathrm{l}$ of water. $5 \mu \mathrm{M}$ of random hexamer oligonucleotides (Life Technologies, Carlsbad, CA) was added to $10 \mu \mathrm{l}$ of the eluate and incubated for 5 minutes at $65^{\circ} \mathrm{C} .5 \mu \mathrm{l}$ of the RNA-random hexamer mix was added to $200 \mathrm{U}$ of MMLV reverse transcriptase (Life Technologies) and cDNA was synthesized using the cycling parameters, $25^{\circ} \mathrm{C}$ for $10 \mathrm{~min}, 42^{\circ} \mathrm{C}$ for $1 \mathrm{hr}$. cDNA was subsequently purified using the MinElute ${ }^{\circ}$ Reaction Cleanup Kit. cDNA systhesis yielded a DNA concentration below the limit of detection ( $<0.2 \mathrm{ng} / \mu \mathrm{l}$ as determined by Qubit (Life Technologies) analysis). These samples were of insufficient quantity to generate a quality library preparation as determined by a Bioanalyzer 2100 (Agilent Technologies, Santa Clara, CA).

\section{Preparation of sequencing libraries and sequencing on Illumina HiSEq 2500}

DNA concentration was determined using the Qubit dsDNA HS kit. DNA quantity and quality was further assessed by product size and concentration using a Bioanalyzer 2100. DNA was sheared with an S2 focusedultrasonicator (Covaris, Woburn, MA) to achieve a target size range of fragments of 100-900 bp. The KAPA High Throughput Library Preparation Kit with Standard PCR Library Amplification (KAPA Biosystems, Wilmington, MA) was used to generate Illumina sequencing libraries according to the manufacturer's instructions. Illumina TruSeq adapters with 6 bp indices were used to enable multiplexed sequencing. Indices on adapters were chosen such as to achieve highest discriminating power between individual libraries. All libraries were amplified with seven PCR cycles. This cycle number was chosen based on the 
minimal number of PCR cycles necessary for the sample with lowest input DNA ( $\mathrm{CsCl} 2)$ and used for all samples to avoid differences between samples due to varying cycle numbers. Illumina libraries were subjected to dual size selection using AMPure XP beads (Beckman Coulter) with a target size of 300 to $1000 \mathrm{bp}$. Library quality was measured on a Bioanalyzer 2100 by product size and concentration. All eight samples were sequenced on a single lane on an Illumina HiSeq 2500 (Illumina, San Diego, CA) in paired-end mode. About 14 million 100 bp pairedend reads were generated for each sample. Sequencing reads were demultiplexed based on the $6 \mathrm{bp}$ index integrated in the Illumina TruSeq adapter sequences allowing for one mismatch using the CASAVA software provided by Illumina.

\section{Read mapping and quantification}

Raw reads were mapped against a set of reference genomes to quantify reads per organism. The following reference genomes were used (NCBI accession numbers if not otherwise noted): bacteriophages, M13 (JX412914.1), P22 (AF217253.1 and AB426868.1), $\$ 6$ (M17461.1, M17 462.1 and M12921.1), T3 (KC960671.1), T7 (NC_0016 04.1) and $\Phi \mathrm{VPE} 25$ (unpublished, available upon request); bacteria, L. monocytogenes EGD-e (AL591824.1), B. thetaiotaomicron VPI-5482 (AE015928.1 and AY171301.1), E. coli BL21(DE3) (NC_012971.2), S. enterica subsp. enterica serovar Typhimurium str. LT2 (NC_003197.1 and NC_003277.1), P. syringae pv. phaseolicola 1448A (CP0 00058.1, CP000059.1 and CP000060.1) and Enterococcus faecalis V583 (NC_004668.1, NC_004669.1, NC_004671.1 and NC_004670.1); phiX174 used as an internal Illumina control (J02482.1); the mouse reference genome (mm10) and the human reference genome (hg38) were downloaded through the UCSC Genome Browser [55].

Reads were mapped onto all reference genomes in parallel using the BBSplit tool, which is part of the BBMap short read aligner tool set (Version 32.15) [56]. The 'ambig2' parameter was set to 'split', resulting in reads that map to more than one reference genome being written into separate files for ambiguous reads. Read mapping statistics for each reference genome were generated by setting the 'refstats' parameter. Additionally the following default parameters were used by the program: match $=$ long, fastareadlen $=500$, minapproxhits $=2$, minratio $=0.9$, maxindel $=20$, trim $=$ both, untrim $=$ true. Reads that did not map to any of the references were output into separate FASTQ files with unmapped reads.

\section{Mouse read mapping visualization}

To visualize the distribution of reads mapping to the mouse reference genome, BAM read alignment files were generated using BBMap. BAM files plus the mouse reference genome were then loaded into the Integrative
Genomics Viewer (Version 2.3.34) [34,57] and read mapping along all chromosomes was inspected visually.

\section{Trimming, assembly and extraction of phage contigs}

To determine how well phage genomes could be reconstructed from the metagenomes, we assembled the metagenomes and then checked for the number and size of phage contigs. We trimmed the raw reads using the 'nesoni clip' tool from the Nesoni high-throughput sequencing data analysis toolset (Version 0.114, http://www.vicbioinformatics.com/software.nesoni.shtml) for Illumina adapters and a minimum quality of 2 . Additionally, we removed the first 9 and last 5 bases from each read. Read quality statistics were checked before and after trimming with the FastQC tool (Version 0.10.1) [58]. Trimmed reads were error corrected with the BayesHammer tool [59] integrated in the SPAdes pipeline and then assembled using the SPAdes assembler (Version 3.0.0) $[60,61]$ in multi-cell mode using k-mer lengths of 21,33, 55 and 77. We searched the resulting assemblies for phage contigs by querying the assemblies with the phage reference genomes using BLASTN (Version 2.2.29+) [62,63].

\section{Availability of supporting data}

The data sets supporting the results of this article are available in the European Nucleotide Archive, PRJEB6941, http://www.ebi.ac.uk/ena/data/view/PRJEB6941.

\section{Additional file}

Additional file 1: Read mapping details and phage enumeration comparison. Table S1. Percent unambiguously mapped reads. Table S2. Percent unambiguously mapped reads normalized to genome size. Table S3. Details on read mapping for sample FD1. Table S4. Details on read mapping for sample FD2. Table S5. Details on read mapping for sample DTT1. Table S6. Details on read mapping for sample DTT2. Table S7. Details on read mapping for sample CsCl1. Table S8. Details on read mapping for sample $\mathrm{CsCl}$. Table S9. Details on read mapping for sample MG1. Table S10. Details on read mapping for sample MG2. Table S11. Plaque forming unit (PFU) count versus virus-like particle (VLP) count for dsDNA phages in phage stocks used to prepare the artificial microbiome sample.

\section{Abbreviations}

CFU: Colony forming unit; dsDNA: Double-stranded DNA; ssDNA: Singlestranded DNA; dsRNA: Double-stranded RNA; MDA: Multiple displacement amplification; PFU: Plaque forming unit; VLP: Virus-like particle.

\section{Competing interests}

The authors declare that they have no competing interests.

\section{Authors' contributions}

MK conceived the study, designed the experiments, cultured and purified bacteriophages, performed all data analyses, and wrote the manuscript. LH conceived the study and helped write the manuscript. BD conceived the study, designed the experiments, cultured the bacterial strains, cultured and purified bacteriophages, ran the purification procedures on the artificial microbiota sample, performed VLP enumerations and helped write the manuscript. All authors read and approved the final manuscript. 


\section{Acknowledgements}

We thank Cassie Behrendt and Tess Leal for assistance with mice. We also thank Vanessa Schmid and the members of the UT-Southwestern Next Generation Sequencing Facility. We are grateful to Paul Turner and Daniel Goldhill for generously providing $\phi 6$ and P. phaseolica HB10Y, Brian Bushnel from the Joint Genome Institute for help using the BBMap tool, and Harald Gruber for helpful suggestions on the bioinformatic analyses. We thank Bruce Beutler of the UT-Southwestern Center for the Genetics of Host Defense for use of the Ultracentrifuge. We also thank the three anonymous reviewers for detailed and thoughtful feedback that helped improve this manuscript significantly. This work was funded by NIH R01 DK070855 (LVH), a Burroughs Wellcome Foundation New Investigators in the Pathogenesis of Infectious Diseases Award (L.V.H.), the Howard Hughes Medical Institute $(\mathrm{LVH})$, and NIH K01 DK102436 (BAD).

\section{Author details}

${ }^{1}$ Department of Immunology, University of Texas Southwestern Medical Center, Dallas, TX 75390, USA. ${ }^{2}$ The Howard Hughes Medical Institute, University of Texas Southwestern Medical Center, Dallas, TX 75390, USA. ${ }^{3}$ Current address: Department of Geoscience, University of Calgary, Calgary, AB T2N 1 N4, Canada.

\section{Received: 24 August 2014 Accepted: 24 December 2014}

Published online: 22 January 2015

\section{References}

1. Bäckhed F, Ley RE, Sonnenburg JL, Peterson DA, Gordon J. Host-bacterial mutualism in the human intestine. Science. 2005;307:1915-20.

2. Wohlgemuth S, Loh G, Blaut M. Recent developments and perspectives in the investigation of probiotic effects. Int J Med Microbiol. 2010;300:3-10.

3. Tremaroli $V$, Bäckhed F. Functional interactions between the gut microbiota and host metabolism. Nature. 2012;489:242-9.

4. Qin J, Li R, Raes J, Arumugam M, Burgdorf KS, Manichanh C, et al. A human gut microbial gene catalogue established by metagenomic sequencing. Nature. 2010;464:59-65

5. Hehemann J-H, Correc G, Barbeyron T, Helbert W, Czizek M, Michel G. Transfer of carbohydrate-active enzymes from marine bacteria to Japanese gut microbiota. Nature. 2010;464:908-12.

6. Muegge BD, Kuczynski J, Knights D, Clemente JC, González A, Fontana L, et al. Diet drives convergence in gut microbiome functions across mammalian phylogeny and within humans. Science. 2011;332:970-4.

7. Reyes A, Haynes M, Hanson N, Angly FE, Heath AC, Rohwer F, et al. Viruses in the faecal microbiota of monozygotic twins and their mothers. Nature. 2010;466:334-8.

8. Minot S, Sinha R, Chen J, Li H, Keilbaugh SA, Wu GD, et al. The human gut virome: inter-individual variation and dynamic response to diet. Genome Res. 2011;21:1616-25.

9. Handley Scott A, Thackray Larissa B, Zhao G, Presti R, Miller Andrew D, Droit $L$, et al. Pathogenic simian immunodeficiency virus infection is associated with expansion of the enteric virome. Cell. 2012;151:253-66.

10. Duerkop BA, Clements CV, Rollins D, Rodrigues JLM, Hooper LV. A composite bacteriophage alters colonization by an intestinal commensal bacterium. Proc Natl Acad Sci USA. 2012;109:17621-6.

11. Duerkop BA, Hooper LV. Resident viruses and their interactions with the immune system. Nat Immunol. 2013;14:654-9.

12. Lengeling A, Mahajan A, Gally DL. Bacteriophages as pathogens and immune modulators? mBio. 2013;4:e00868-13.

13. Barr JJ, Auro R, Furlan M, Whiteson KL, Erb ML, Pogliano J, et al. Bacteriophage adhering to mucus provide a non-host-derived immunity. Proc Natl Acad Sci USA. 2013;110:10771-6.

14. Reyes A, Wu M, McNulty NP, Rohwer FL, Gordon Jl. Gnotobiotic mouse model of phage-bacterial host dynamics in the human gut. Proc Natl Acad Sci USA. 2013;110:20236-41.

15. Reyes A, Semenkovich NP, Whiteson K, Rohwer F, Gordon Jl. Going viral: next-generation sequencing applied to phage populations in the human gut. Nat Rev Microbiol. 2012;10:607-17.

16. Hurwitz BL, Deng L, Poulos BT, Sullivan MB. Evaluation of methods to concentrate and purify ocean virus communities through comparative, replicated metagenomics. Environ Microbiol. 2013;15:1428-40.
17. Solonenko S, Ignacio-Espinoza J, Alberti A, Cruaud C, Hallam S, Konstantinidis K, et al. Sequencing platform and library preparation choices impact viral metagenomes. BMC Genomics. 2013;14:320.

18. Duhaime MB, Deng L, Poulos BT, Sullivan MB. Towards quantitative metagenomics of wild viruses and other ultra-low concentration DNA samples: a rigorous assessment and optimization of the linker amplification method. Environ Microbiol. 2012;14:2526-37.

19. Yilmaz S, Allgaier M, Hugenholtz P. Multiple displacement amplification compromises quantitative analysis of metagenomes. Nat Meth. 2010;7:943-4.

20. Marine R, McCarren C, Vorrasane V, Nasko D, Crowgey E, Polson S, et al. Caught in the middle with multiple displacement amplification: the myth of pooling for avoiding multiple displacement amplification bias in a metagenome. Microbiome. 2014;2:3.

21. Ellegaard KM, Klasson $L$, Andersson SGE. Testing the reproducibility of multiple displacement amplification on genomes of clonal endosymbiont populations. PLOS ONE. 2013;8:e82319.

22. Campbell A. The future of bacteriophage biology. Nat Rev Genet. 2003:4:471-7.

23. Thurber RV, Haynes M, Breitbart M, Wegley L, Rohwer F. Laboratory procedures to generate viral metagenomes. Nat Protocols. 2009;4:470-83.

24. Yamamoto KR, Alberts BM, Benzinger R, Lawhorne L, Treiber G. Rapid bacteriophage sedimentation in the presence of polyethylene glycol and its application to large-scale virus purification. Virology. 1970;40:734-44.

25. Sambrook J, Fritsch EF, Maniatis T. Bacteriophage I growth, purification, and DNA extraction. In: Nolan C, editor. Molecular Cloning. Vol. 1. 2nd ed. Cold Spring Harbor, NY: Cold Spring Harbor Laboratory Press; 1989.

26. Lawrence JE, Steward GF. Purification of viruses by centrifugation. In: Wilhelm SW, Weinbauer MG, editors. Manual of aquatic viral ecology. Suttle CA: ASLO; 2010. p. 166-81.

27. Brum JR, Steward GF. Physical fractionation of aquatic viral assemblages Limnol Oceanograph: Methods. 2011;9:150-63.

28. Kirchman DL. Ecology of viruses. In: Processes in microbial ecology. Croydon, UK: CPI Group; 2012.

29. Labrie SJ, Samson JE, Moineau S. Bacteriophage resistance mechanisms. Nat Rev Microbiol. 2010;8:317-27.

30. Gansauge M-T, Meyer M. Single-stranded DNA library preparation for the sequencing of ancient or damaged DNA. Nat Protoc. 2013:8:737-48.

31. Ackermann HW. 5500 Phages examined in the electron microscope. Arch Virol. 2007;152:227-43.

32. Cordova A, Deserno M, Gelbart WM, Ben-Shaul A. Osmotic shock and the strength of viral capsids. Biophys J. 2003;85:70-4.

33. Erlwein O, Robinson MJ, Dustan S, Weber J, Kaye S, McClure MO. DNA extraction columns contaminated with murine sequences. PLoS ONE. 2011;6:e23484.

34. Integrative Genomics Viewer. [http://www.broadinstitute.org/igv/home]

35. Allander T, Emerson SU, Engle RE, Purcell RH, Bukh J. A virus discovery method incorporating DNase treatment and its application to the identification of two bovine parvovirus species. Proc Natl Acad Sci USA. 2001;98:11609-14

36. Croucher NJ, Fookes MC, Perkins TT, Turner DJ, Marguerat SB, Keane T, et al. A simple method for directional transcriptome sequencing using Illumina technology. Nucleic Acids Res. 2009;37:e148.

37. Kuhn H, Frank-Kamenetskii MD. Template-independent ligation of single-stranded DNA by T4 DNA ligase. FEBS J. 2005;272:5991-6000.

38. Kim K-H, Chang H-W, Nam Y-D, Roh SW, Kim M-S, Sung Y, et al. Amplification of uncultured single-stranded DNA viruses from rice paddy soil. Appl Environ Microbiol. 2008;74:5975-85.

39. Haible D, Kober $\mathrm{S}$, Jeske $\mathrm{H}$. Rolling circle amplification revolutionizes diagnosis and genomics of geminiviruses. J Virol Methods. 2006;135:9-16.

40. Tucker KP, Parsons R, Symonds EM, Breitbart M. Diversity and distribution of single-stranded DNA phages in the North Atlantic Ocean. ISME J. 2011;5:822-30.

41. Andrews-Pfannkoch C, Fadrosh DW, Thorpe J, Williamson SJ. Hydroxyapatitemediated separation of double-stranded DNA, single-stranded DNA, and RNA genomes from natural viral assemblages. Appl Environ Microbiol. 2010;76:5039-45.

42. Goodrich Julia K, Di Rienzi Sara C, Poole Angela C, Koren O, Walters William A, Caporaso JG, et al. Conducting a microbiome study. Cell. 2014;158:250-62.

43. Cash HL, Whitham CV, Behrendt CL, Hooper LV. Symbiotic bacteria direct expression of an intestinal bactericidal lectin. Science. 2006;313:1126-30. 
44. Turner P, McBride R, Duffy S, Montville R, Wang L-S, Yang Y, et al. Evolutionary genomics of host-use in bifurcating demes of RNA virus phi-6. BMC Evol Biol. 2012;12:153.

45. Paulsen IT, Banerjei L, Myers GSA, Nelson KE, Seshadri R, Read TD, et al. Role of mobile DNA in the evolution of vancomycin-resistant Enterococcus faecalis. Science. 2003;299:2071-4.

46. Glaser P, Frangeul L, Buchrieser C, Rusniok C, Amend A, Baquero F, et al. Comparative genomics of Listeria species. Science. 2001;294:849-52.

47. Xu J, Bjursell MK, Himrod J, Deng S, Carmichael LK, Chiang HC, et al. A genomic view of the human-Bacteroides thetaiotaomicron symbiosis. Science. 2003:299:2074-6

48. Hershey AD, Kalmanson G, Bronfenbrenner J. Quantitative methods in the study of the phage-antiphage reaction. J Immunol. 1943;46:267-79.

49. Kim M-S, Park E-J, Roh SW, Bae J-W. Diversity and abundance of singlestranded DNA viruses in human feces. Appl Environ Microbiol. 2011;77:8062-70.

50. Colson P, Fancello L, Gimenez G, Armougom F, Desnues C, Fournous G, et al. Evidence of the megavirome in humans. J Clin Virol. 2013;57:191-200.

51. Abeles SR, Pride DT. Molecular bases and role of viruses in the human microbiome. J Mol Biol. 2014;426:3892-906.

52. King AMQ, Lefkowitz E, Adams MJ, Carstens EB, editors. Virus taxonomy: Ninth report of the international committee on taxonomy of viruses. London, UK: Elsevier Academic Press; 2012

53. Kovatcheva-Datchary P, Tremaroli V, Bäckhed F. The gut microbiota. In: Rosenberg E, DeLong EF, Lory S, Stackebrandt E, Thompson FL, editors. The prokaryotes - human microbiology. 4th ed. Berlin Heidelberg: Springer; 2013.

54. Rappe MS, Connon SA, Vergin KL, Giovannoni SJ. Cultivation of the ubiquitous SAR11 marine bacterioplankton clade. Nature. 2002:418:630-3.

55. UCSC Genome Browser. [https://genome.ucsc.edu/]

56. BBMap short read aligner, and other bioinformatic tools. [http://sourceforge. net/projects/bbmap/]

57. Thorvaldsdóttir H, Robinson JT, Mesirov JP. Integrative Genomics Viewer (IGV): high-performance genomics data visualization and exploration. Brief Bioinform. 2013;14:178-92.

58. FastQC: a quality control tool for high throughput sequence data. [http://www.bioinformatics.babraham.ac.uk/projects/fastqc/)]

59. Nikolenko S, Korobeynikov A, Alekseyev M. BayesHammer: Bayesian clustering for error correction in single-cell sequencing. BMC Genomics. 2013;14:S7.

60. SPAdes assembly pipeline. [http://bioinf.spbau.ru/spades]

61. Bankevich A, Nurk S, Antipov D, Gurevich AA, Dvorkin M, Kulikov AS, et al. SPAdes: a new genome assembly algorithm and its applications to singlecell sequencing. J Comput Biol. 2012;19:455-77.

62. BLAST+. [ftp://ftp.ncbi.nlm.nih.gov/blast/executables/blast+/]

63. Camacho C, Coulouris G, Avagyan V, Ma N, Papadopoulos J, Bealer K, et al. BLAST+: architecture and applications. BMC Bioinf. 2009;10:421.

\section{Submit your next manuscript to BioMed Central and take full advantage of:}

- Convenient online submission

- Thorough peer review

- No space constraints or color figure charges

- Immediate publication on acceptance

- Inclusion in PubMed, CAS, Scopus and Google Scholar

- Research which is freely available for redistribution 